\title{
EOQ Model under Permissible Delay in Payments and Inflation with Weibull Decay and Power Pattern Demand
}

\author{
K. Srinivasa Rao ${ }^{1}$, K.Nagendra Kumar ${ }^{2}$, B.Muniswamy ${ }^{3}$ \\ Department of Statistics ${ }^{1,2,3}$, Andhra University, Visakhapatnam, India \\ Email: ksraoau@yahoo.co.in ${ }^{1}, \underline{\text { nagendra.vij@gmail.com, munistats@gmail.com }}$
}

\begin{abstract}
Recently much work has reported regarding EOQ models for deteriorating items with delay in payments. Most of these papers considered that there is no change of money value over time. But money value changes due to inflation. Hence, in this paper we develop and analyze an EOQ model for deteriorating items with the assumption that delay in payments is allowed under inflation. It is assumed that the life time of the commodity is random and follows a Weibull distribution. It is further assumed that the demand rate is time dependent and follows power pattern, with different values of indexing parameter the demand rate may increase or decrease or remains constant. Using the differential equations the instantaneous state of inventory at a given time is obtained explicitly. With plausible cost considerations under inflation the total cost function over the horizon is derived. The net profit rate function is also obtained. By maximizing the net profit rate function the optimal ordering and price policies are obtained. It is observed through sensitivity analysis that the deterioration distribution parameters, demand rate parameters and inflation rate have significant influence on the optimal operating policies of the model. The permissible delay in payments affects the ordering quantity and cycle time. This model also includes some of the earlier models as particular cases for specific values of the parameters.
\end{abstract}

Keywords: EOQ Model, Weibull decay, Delay in payments, inflation rate, time dependent deterioration.

\section{INTRODUCTION}

Economic Order Quantity models play an important role in planning several systems at market yards, warehouses, production processes, civil supply departments. The EOQ models for deteriorating items are required for developing optimal ordering and pricing policies of the organizations dealing with the goods which are subject to decay, perishable, damage, obsolete and evaporation. The rate of deterioration has significance influence on developing optimal policies of the systems (Srinivasarao et al.,(2010)). Much work has been reported in literature regarding EOQ models for deteriorating items. Nahmias. S(1978), Raafat (1991), Giri and Goyal(2001), Ruxian and L.i, et al. (2010) Dhir Singh and Singh(2018) have presented an elegant review on inventory models for deteriorating items Ghare and Scharder(1963), Shah and Jailwal (1977), Cohen(1976), Aggarwal(1978), Dave and Shah(1982), pal (1990), Giri and Chaudhari(1980), Thadikulla(1978) covert and Philip(1973), Philip(1974), Goyal and Agarwal(1980), Venkatasubbaiah (1999), Nirupama Devi et. al. (2004) and others have assumed the variable rate of deterioration. In all these models, it is assumed that the payments must be made to the supplier immediately after receiving the items.
However, in practice, the supplier allows a certain fixed period for setting the account and does not charge any interest to the retailer during this period. Goyal (1985) developed an EOQ model under permissible delay in payments. Aggarwal and Jaggi (1995) extended Goyal's(1985) model to consider the deteriorating items. Jamal et. al.(1997) further generalized Aggarwal and Jaggi's (1995) model to allow shortages. Other interesting and relevant papers related to the delay in payments are Sarkeret et al. (2001), Chung and Liao (2004), Ouyang et. al., (2005). Ouyang et al. (2002) extended Goyal's(1985) model to consider cash discount and delay in payments and established an analytical closed form solution to the problem. Liang, Yuh Ouyang, Kun-Shan Wu and ChihTeyang (2006), Jui-Jung Liao (2007), Chandra K. Jaggi, S.K.Goel (2008) and Chao Kuei Huang (2009), Horng-Jinh et al. (2002), R. Amutha et al. (2013), H.S. Shukla et al. (2015), R.P. Tripathi et al. (2016), Jyothi (2018) and others have also studied inventory models with delay in payments. In all these models they assumed that there is no change of money value over time.

However, the purchaser may invest the money on the stock market or to develop new products, and get a return from the investment which may be higher than interest changes. Thus, 


\section{International Journal of Research in Advent Technology, Vol.7, No.1, January 2019 \\ E-ISSN: 2321-9637 \\ Available online at www.ijrat.org}

the purchaser may not pay the supplier at the end of the credit period, instead, he or she will invest the money until the interest payable to the supplier is larger than the interest earned. Consequently, it is more radical for the purchaser either to pay the supplier at the end of the credit period or to incur interest charges on the unpaid balance for the overdue period. This consideration leads to consider the current large scale inflation in world economy and its impact on the optimal policies of the inventory systems. From the financial point inflation is generally associated with rapidly raising prices which causes or caused by a decline in the purchasing power of money which varies or rather depends on time.

BuzaCott (1975), Bierman and Thomas (1977) and Misra (1979) analyzed inventory decision under inflation for the EOQ model, Brahmhatt (1982) developed EOQ model under a variable rate of inflation. Ravi Gor and Nita H Shah (2006), ManusriBasu, Samiran Senpaati and Kanailal Banergjee (2006), Jayanta Kumar Dey, Shyamal Kumar Mondla Manoranjan Maiti(2008) have developed EOQ models for deteriorating items under permissible delay in payments under inflation. They assumed that the rate of deterioration is constant. But in may inventory situations the rate of deterioration is a variable depending on time. Raman Patel et al. (2017) have studied an inventory model with different deterioration rates with shortages, time and price dependent demand under inflation and permissible delay in payments. They considered that the rate of deterioration is $\theta$ during $\mu_{1} \leq t \leq \mu_{2}$ and $\theta t$ when $\mu_{2} \leq t \leq t_{0}$ and $0<\theta<1$. With the same assumption on deterioration, Shital S Patel (2017) has studied the inventory model under inflation and permissible delay in payments for imperfect quality items.

In may practical situations the deterioration is time dependent and variable. In addition to this it is stochastic due to various random factors influencing the life time. The variable rate of deterioration can be well characterized by Weibull distribution. The Weibull decay can include increasing or decreasing or constant rates of deterioration. Very little work has been reported in literature regarding inventory models under permissible delay in payments and inflation having Weibull rate of decay except the workless of Susheel Kumar et al. (2013) who developed an inventory model for Weibull deteriorating items with constant demand and under permissible delay in payments. Sanjay Kumar and Neeraj Kumar (2016) have studied an inventory model with stock dependent demand and Weibull rate of deterioration having permissible delay in payments under inflation using genetic algorithm. However, they assumed that the demand is either constant or stock dependent.

But in many market yards it is observed that the demand is time dependent and follows a power pattern. Hence, in this paper we develop and analyze an EOQ model for deteriorating items under permissible delay in payments under inflation with demand as function of time and follows power pattern. The power pattern demand can include increasing/ decreasing/ constant rates of demand for different values of index parameter. Using the differential equations the instantaneous state of inventory at given time ' $t$ ' is obtained. With suitable cost considerations, the total cost over the horizon is derived. By maximizing the net profit rate function the optimal ordering policies are obtained. The behavior of the model with respect to the change in parameters and costs is also analyzed. The effect of inflation is studied. The model is useful for developing the optimal ordering policies of systems at vegetable markets and market yards.

\section{ASSUMPTIONS:}

Consider an inventory system with the following assumptions for developing the EOQ model:

i) The life time of the commodity is random and follows a Weibull distribution. Hence, the instantaneous rate of deterioration $h(t)$ is

$$
h(t)=\alpha \beta t^{\alpha-1}, t>0
$$

ii) The demand is time dependent and follows a power pattern demand which is of the form

$$
R(t)=a-b_{1}\left[\frac{\theta t^{\frac{1}{n}-1}}{n T^{\frac{1}{n}}}\right]
$$

where, $\mathrm{a}$ is fixed demand, $a>0, \mathrm{~b}$ is a demand parameter, $\mathrm{b}>0$ and $a>b, \theta$ is the total demand during the cycle period, $\mathrm{n}$ is the index parameter. iii) Rate of inflation and its values is $r, 0<r<1$.

iv) Shortages are not allowed.

v) Lead time is zero.

iv) During the permissible delay period (M), the account is not settled, the generated sales revenue is deposited in an interest-bearing account. At the end of the trade credit period, the customer pays off for all the units ordered.

vii)There is no repair or replacement of the deteriorated units during the cycle time $(\mathrm{T})$. 


\section{INVENTORY MODEL}

Consider an inventory system in which the inventory level is $\mathrm{Q}$ at time $\mathrm{t}=0$. During $(0, \mathrm{~T})$ the inventory level gradually decreases due to demand and deterioration. Since no shortages are allowed at time $\mathrm{T}$ as and when the inventory level reaches zero the stock is replenished instantaneously. The schematic diagram representing the inventory level is shown in Figure-1

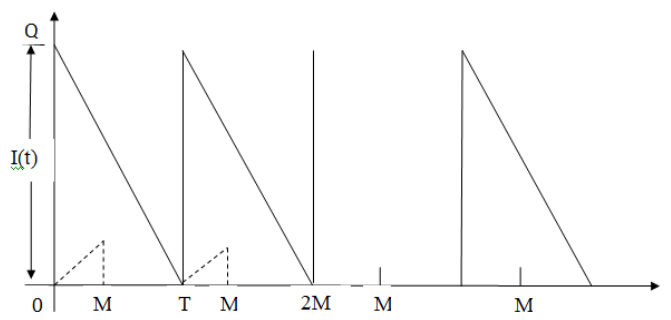

Figure -1 Schematic diagram representing the inventory level.

Let $I(t)$ is the inventory level at time $t$ then the differential equations governing the inventory level at time ' $\mathrm{t}$ ' is

$$
\frac{d}{d t} I(t)+h(t) I(t)=-R(T), \quad 0 \leq t \leq T
$$

This implies

$$
\frac{d}{d t} I(t)+\left[\alpha \beta t^{\alpha-1}\right] I(t)=-\left[a-b_{1}\left(\frac{\theta t^{\frac{1}{n}-1}}{n T^{\frac{1}{n}}}\right)\right]
$$

Solving the equation (3.1) we get

$$
\mathrm{I}(\mathrm{t})=e^{-\beta t^{\alpha}} \int_{t}^{T}\left[a-b_{1}\left(\frac{\theta t^{\frac{1}{n}-1}}{n T^{\frac{1}{n}}}\right)\right] e^{\beta t^{\alpha}} d t
$$

Therefore the inventory level $\mathrm{I}(\mathrm{t})$ at any time ' $\mathrm{t}$ ' is

$\mathrm{I}(\mathrm{t})=e^{\beta t^{\alpha}} \int_{t}^{T}\left[a-b_{1}\left(\frac{\theta t^{\frac{1}{n}-1}}{n T^{\frac{1}{n}}}\right)\right] e^{\beta t^{\alpha}} d t, 0 \leq t \leq T$

Using boundary condition $\mathrm{I}(0)=\mathrm{Q}$, one can get the ordering quantity as

$$
Q=\int_{0}^{T}\left[a-b_{1}\left(\frac{\theta t^{\frac{1}{n}-1}}{n T^{\frac{1}{n}}}\right)\right] e^{\beta t^{\alpha}} d t, 0 \leq t \leq T
$$

Since the length of time internals are all the same, we have

$$
\mathrm{I}(\mathrm{jT}+\mathrm{t})=e^{-\beta t^{\alpha}} \int_{0}^{T}\left[a-b_{1}\left(\frac{\theta t^{\frac{1}{n}-1}}{n T^{\frac{1}{n}}}\right)\right] e^{\beta t^{\alpha}} d t,
$$

where $0 \leq J \leq n-1,0 \leq t \leq T$

\section{OPTIMAL ORDERING AND PRICING POLICIES OF THE MODEL:}

In this section, the total cost function is obtained as a sum of cost of placing orders (OC), Cost of deteriorated units (CD), cost of carrying inventory(IHC), interest earned. Each cost component is computed as follows:

The cost of placing orders (OC) is

$$
\begin{aligned}
\mathrm{OC}=\mathrm{A}(0)+\mathrm{A} & (\mathrm{T})+\mathrm{A}(2 \mathrm{~T}) \\
& +\mathrm{A}(3 \mathrm{~T})+\ldots \ldots+\mathrm{A}((\mathrm{n}-1) \mathrm{T}) \\
& =\frac{\mathrm{A}\left(\mathrm{e}^{\mathrm{rH}}-1\right)}{\left(\mathrm{e}^{\mathrm{rH}}-1\right)}
\end{aligned}
$$

The cost of deteriorated units (CD) is

$$
\mathrm{CD}=\sum_{\mathrm{j}=0}^{\mathrm{n}-1} \mathrm{C} \mathrm{e}^{\mathrm{rit}}\left[\mathrm{Q}-\int_{0}^{\mathrm{m}}\left[a-b_{1}\left(\frac{\theta t^{\frac{1}{n}-1}}{n T^{\frac{1}{n}}}\right)\right] \mathrm{dt}\right],
$$

where, $\mathrm{Q}$ is as given in equation (4).

Therefore,

$$
\mathrm{CD}=\mathrm{C}\left[\int_{0}^{\mathrm{T}}\left[a-b_{1}\left(\frac{\theta t^{\frac{1}{n}-1}}{n T^{\frac{1}{n}}}\right)\right] e^{\beta t^{\alpha}} d t-a T+b_{1} \theta\right]\left(\frac{e^{r H}-1}{e^{r T}-1}\right)
$$

The cost of carrying inventory (IHC) is

$$
I H C=h \sum_{j=0}^{n-1} C(j T) \int_{0}^{T} I(j T+t) d t
$$

Substituting the equation (3) in the IHC one can get

$$
\mathrm{IHC}=\mathrm{Ch}\left[\int_{0}^{\mathrm{T}} e^{\beta t^{\alpha}}\left[\int_{0}^{t}\left[a-b_{1}\left(\frac{\theta t^{\frac{1}{n}-1}}{n T^{\frac{1}{n}}}\right)\right] e^{\beta t^{\alpha}} d t\right] \mathrm{dt}\left(\frac{e^{r H}-1}{e^{r T}-1}\right)\right.
$$

For computing the interest charged and earned. There are two possibilities based on the customer's choice. Cost of inventory changes (IC) for unsold items at the initial time or after the permissible delay period ' $M$ ' and interest earned (IE) from the sales revenue during the permissible delay period.

\section{Case(i) Replenishment level ' $T$ ' is larger than or equal to i.e., $M \leq T$}

Interest Charged in $(\mathrm{O}, \mathrm{H})$ is

$$
\begin{aligned}
& \mathrm{IC}_{1}=\mathrm{I}_{\mathrm{C}} \sum_{\mathrm{J}=0}^{\mathrm{n}-1} \mathrm{C}(\mathrm{jT}) \int_{\mathrm{m}}^{\mathrm{T}} \mathrm{I}(\mathrm{j} \mathrm{T}+\mathrm{t}) \mathrm{dt} \\
& =\mathrm{CI}_{\mathrm{c}}\left[\int_{m}^{T} e^{-\beta t^{\alpha}}\left[\int_{0}^{t}\left[a-b_{1}\left(\frac{\theta t^{\frac{1}{n}-1}}{n T^{\frac{1}{n}}}\right)\right]\right] e^{\beta t^{\alpha}} d t\right] d t\left(\frac{e^{r H}-1}{e^{r T}-1}\right)
\end{aligned}
$$

Interest earned in $(\mathrm{O}, \mathrm{H})$ is

$$
\begin{aligned}
\mathrm{IE}_{1} & =\mathrm{I}_{e} \sum_{\mathrm{j}=0}^{\mathrm{n}-1} \mathrm{P}(\mathrm{jT}) \int_{0}^{\mathrm{m}}\left[a-b_{1}\left(\frac{\theta t^{\frac{1}{n}-1}}{n T^{\frac{1}{n}}}\right)\right] \mathrm{tdt} \\
& =\mathrm{pI}_{e}\left[\int_{0}^{\mathrm{M}} \text { at } \mathrm{dt}-\mathrm{b}_{1} \theta \int_{0}^{\mathrm{M}} \frac{\mathrm{t}^{\frac{1}{n}}}{\mathrm{n} \mathrm{T}^{\frac{1}{n}}} \mathrm{dt}\right]\left[\frac{e^{r H}-1}{e^{r T}-1}\right]
\end{aligned}
$$




$$
\begin{aligned}
& =\mathrm{pI}_{e}\left[\left(\frac{\mathrm{at}^{2}}{2}\right)_{0}^{\mathrm{M}}-\frac{\mathrm{b}_{1} \theta}{\mathrm{nT}^{\frac{1}{n}}}\left(\frac{\mathrm{t}^{\frac{1}{n}+1}}{\frac{1}{\mathrm{n}}+1}\right)_{0}^{\mathrm{M}}\right]\left[\frac{e^{r H}-1}{e^{r T}-1}\right] \\
& =\mathrm{pI}_{e}\left[\frac{\mathrm{aM}^{2}}{2}-\frac{\mathrm{b}_{1} \theta}{\mathrm{nT}^{\frac{1}{n}}}\left(\frac{\mathrm{M}^{\frac{1}{\mathrm{n}}+1}}{\frac{1}{\mathrm{n}}+1}\right)\right]\left[\frac{e^{r H}-1}{e^{r T}-1}\right] \\
& =\mathrm{pI}_{e}\left[\frac{\mathrm{aM}^{2}}{2}-\frac{\mathrm{b}_{1} \theta}{\mathrm{nT}^{\frac{1}{n}}}\left(\frac{\mathrm{nM}^{\frac{\mathrm{n}+1}{\mathrm{n}}}}{\mathrm{n}+1}\right)\right]\left[\frac{e^{r H}-1}{e^{r T}-1}\right]
\end{aligned}
$$

The total cost per unit time cover $(\mathrm{O}, \mathrm{H})$ is

$$
\begin{gathered}
\mathrm{TC}=\mathrm{OC}+\mathrm{CD}+\mathrm{IHC}+\mathrm{IC}_{1}-\mathrm{IE}_{1} \\
\mathrm{TC}(\mathrm{p}, \mathrm{T})=\frac{1}{\mathrm{~T}}\left[\mathrm{~A}+\mathrm{C}\left[\int_{0}^{\mathrm{T}}\left[\mathrm{a}-b_{1}\left[\frac{\theta t^{\frac{1}{n}-1}}{n T^{\frac{1}{n}}}\right] e^{\beta t^{\alpha}} d t-a T+b \theta\right]\right.\right. \\
+\mathrm{Ch}\left[\int_{\mathrm{m}}^{\mathrm{T}} e^{-\beta t^{\alpha}}\left[\int_{\mathrm{t}}^{\mathrm{T}}\left[\mathrm{a}-b_{1}\left[\frac{\theta t^{\frac{1}{n}-1}}{n T^{\frac{1}{n}}}\right]\right] e^{\beta t^{\alpha}} d t\right]\right] \\
+\mathrm{CI}_{\mathrm{C}}\left[\int_{\mathrm{M}}^{\mathrm{T}} e^{-\beta t^{\alpha}}\left[\int_{\mathrm{t}}^{\mathrm{T}}\left[\mathrm{a}-b_{1}\left[\frac{\theta t^{\frac{1}{n}-1}}{n T^{\frac{1}{n}}}\right]\right] e^{\beta t^{\alpha}} d t\right] d \mathrm{dt}\right. \\
-\mathrm{pI}_{\mathrm{e}}\left[\frac{\mathrm{aM}^{2}}{2}-\frac{\mathrm{b}_{1} \theta \mathrm{M}^{\frac{\mathrm{n}+1}{\mathrm{n}}}}{T^{\frac{1}{n}}(n+1)}\right]\left[\frac{e^{r H}-1}{e^{r T}-1}\right]
\end{gathered}
$$

The net profit rate function is the difference of gross revenue and total cost per a unit time. The gross revenue per a unit time at time ' $\mathrm{T}$ ' is

$$
=\left(p e^{r T}-C e^{r T}\right)\left(a-\frac{b_{1} \theta}{n T}\right)
$$

The net profit is

$$
\begin{aligned}
& \mathrm{NP}(\mathrm{p}, \mathrm{T})=\left(p e^{r T}-C e^{r T}\right)\left(a-\frac{b_{1} \theta}{n T}\right) \\
& -\frac{1}{\mathrm{~T}}\left[\mathrm{~A}+\mathrm{C}\left[\int_{0}^{\mathrm{T}}\left[\mathrm{a}-b_{1}\left[\frac{\theta t^{\frac{1}{n}-1}}{n T^{\frac{1}{n}}}\right]\right] e^{\beta t^{\alpha}} d t-a T+b \theta\right]\right. \\
& +\mathrm{Ch}\left[\int_{0}^{\mathrm{T}} e^{-\beta t^{\alpha}}\left[\int_{\mathrm{t}}^{\mathrm{T}}\left[\mathrm{a}-b_{1}\left[\frac{\theta t^{\frac{1}{n}-1}}{n T^{\frac{1}{n}}}\right]\right] e^{\beta t^{\alpha}} d t\right] d t\right] \\
& +\mathrm{CI}_{\mathrm{C}}\left[\int_{\mathrm{M}}^{\mathrm{T}} e^{-\beta t^{\alpha}}\left[\int_{\mathrm{t}}^{\mathrm{T}}\left[\mathrm{a}-b_{1}\left[\frac{\theta t^{\frac{1}{n}-1}}{n T^{\frac{1}{n}}}\right]\right] e^{\beta t^{\alpha}} d t\right] \mathrm{dt}\right] \\
& -\mathrm{pI}_{e}\left[\frac{\mathrm{aM}^{2}}{2}-\frac{\mathrm{b}_{1} \theta \mathrm{M}^{\frac{\mathrm{n}+1}{\mathrm{n}}}}{T^{\frac{1}{n}}(n+1)}\right]\left[\frac{e^{r H}-1}{e^{r T}-1}\right]
\end{aligned}
$$

For obtaining the optimal pricing policies of the model one has to maximize NP ( $p, T)$ with respect to $\mathrm{T}$ and $\mathrm{p}$. The conditions for obtaining optimality are

$$
\begin{array}{r}
\frac{\partial N P}{\partial p}(p, T)=0, \frac{\partial N P}{\partial T}(p, T)=0 \\
\text { and } \mathrm{D}=\left|\begin{array}{ll}
\frac{\partial^{2} N P(p, T)}{\partial p^{2}} & \frac{\partial^{2} N P(p, T)}{\partial p \partial T} \\
\frac{\partial^{2} N P(p, T)}{\partial p \partial T} & \frac{\partial^{2} N P(p, T)}{\partial T^{2}}
\end{array}\right|<0
\end{array}
$$

where, D is determinant of the Hessian Matrix $\frac{\partial N P(p, T)}{\partial p}=0$

$e^{r T}\left(a-\frac{b_{1} \theta}{n T}\right)-\frac{\mathrm{I}_{\mathrm{c}}}{T}\left[\frac{\mathrm{aM}^{2}}{2}-\frac{\mathrm{b}_{1} \theta \mathrm{M}^{\frac{\mathrm{n}+1}{\mathrm{n}}}}{T^{\frac{1}{n}}(n+1)}\right]\left[\frac{e^{r H}-1}{e^{r T}-1}\right]=0$

and $\frac{\partial N P(p, T)}{\partial T}=0$ implies

$$
\begin{aligned}
& \left(p e^{r T} r-C e^{r T} r\right)\left(a-\frac{b_{1} \theta}{n T}\right)+\left(p e^{r T}-C e^{r T}\right)\left(\frac{b_{1} \theta}{n T^{2}}\right) \\
& +\frac{1}{\mathrm{~T}^{2}}\left[\mathrm{~A}+\mathrm{C}\left[\int_{0}^{\mathrm{T}}\left[\mathrm{a}-b_{1}\left[\frac{\theta t^{\frac{1}{n}-1}}{n T^{\frac{1}{n}}}\right]\right] e^{\beta t^{\alpha}} d t-a T+b \theta\right]\right] \\
& +\mathrm{Ch}\left[\int_{0}^{\mathrm{T}} e^{-\beta t^{\alpha}}\left[\int_{\mathrm{t}}^{\mathrm{T}}\left[\mathrm{a}-b_{1}\left[\frac{\theta t^{\frac{1}{n}-1}}{n T^{\frac{1}{n}}}\right]\right] e^{\beta t^{\alpha}} d t\right] \mathrm{dt}\right] \\
& +\mathrm{CI}_{\mathrm{c}}\left[\int_{\mathrm{M}}^{\mathrm{T}} e^{-\beta t^{\alpha}}\left[\int_{\mathrm{t}}^{\mathrm{T}}\left[\mathrm{a}-b_{1}\left[\frac{\theta t^{\frac{1}{n}-1}}{n T^{\frac{1}{n}}}\right]\right] e^{\beta t^{\alpha}} d t\right] \mathrm{dt}\right] \\
& -p \mathrm{I}_{\mathrm{e}}\left[\frac{\mathrm{aM}^{2}}{2}-\frac{\mathrm{b}_{1} \theta \mathrm{M}^{\frac{\mathrm{n}+1}{\mathrm{n}}}}{T^{\frac{1}{n}}(n+1)}\right]\left[\left(\frac{e^{r H}-1}{e^{r T}-1}\right)\right] \\
& -\frac{1}{\mathrm{~T}}\left[\mathrm{~A}+\mathrm{C}\left[\int_{0}^{\mathrm{T}}\left[\mathrm{a}-b_{1}\left[\frac{\theta t^{\frac{1}{n}-1}}{n T^{\frac{1}{n}}}\right]\right] e^{\beta t^{\alpha}} d t-a T+\mathrm{b}_{1} \theta\right]\right. \\
& +\operatorname{Ch}\left[\int_{0}^{\mathrm{T}} e^{-\beta t^{\alpha}}\left[\int_{\mathrm{t}}^{\mathrm{T}}\left[\mathrm{a}-b_{1}\left[\frac{\theta t^{\frac{1}{n}-1}}{n T^{\frac{1}{n}}}\right]\right] e^{\beta t^{\alpha}} d t\right] \mathrm{dt}\right] \\
& +\mathrm{CI}_{\mathrm{c}}\left[\int_{\mathrm{M}}^{\mathrm{T}} e^{-\beta t^{\alpha}}\left[\int_{\mathrm{t}}^{\mathrm{T}}\left[\mathrm{a}-b_{1}\left[\frac{\theta t^{\frac{1}{n}-1}}{n T^{\frac{1}{n}}}\right]\right] e^{\beta t^{\alpha}} d t\right] \mathrm{dt}\right] \\
& -p \mathrm{I}_{\mathrm{e}}\left[\frac{\mathrm{aM}^{2}}{2}-\frac{\mathrm{b}_{1} \theta \mathrm{M}^{\frac{\mathrm{n}+1}{\mathrm{n}}}}{T^{\frac{1}{n}}(n+1)}\right]\left[\frac{r e^{r T}\left(e^{r H}-1\right)}{\left(e^{r T}-1\right)^{2}}\right] \\
& -\frac{1}{T}\left[C\left[a-\frac{b 1 \theta}{n T}\right] e^{\beta t^{\alpha}}-a+\int_{0}^{T} \frac{1}{T^{\frac{n+1}{n}}} \frac{b_{1} \theta t^{\frac{1}{n}-1}}{n T^{\frac{1}{n}}} e^{\beta t^{\alpha}} d t\right] \\
& +C h\left[e^{-\beta t^{\alpha}}\left[a-\frac{b 1 \theta}{n T}\right] \int_{0}^{\mathrm{T}} e^{\beta t^{\alpha}} d t+\int_{0}^{\mathrm{T}} e^{-\beta t^{\alpha}}\left[\int_{t}^{T} \frac{b_{1} \theta t^{\frac{1}{n}-1}}{\mathrm{n}^{2} T^{\frac{1}{n}}} e^{\beta t^{\alpha}} d t\right] \mathrm{dt}\right] \\
& +\mathrm{CI}_{\mathrm{c}}\left[\left(a-\frac{b 1 \theta}{n T}\right) e^{\beta t^{\alpha}} \int_{M}^{T} e^{-\beta t^{\alpha}} d t+\int_{M}^{T} e^{\beta t^{\alpha}}\left[\frac{-b_{1} \theta t^{\frac{1}{n}-1}}{\mathrm{n}^{2} T^{\frac{1}{n}}}\right] d t\right] \\
& -\mathrm{pI}_{\mathrm{e}}\left[\frac{\mathrm{b}_{1} \theta \mathrm{M}^{\frac{\mathrm{n}+1}{\mathrm{n}}}}{T^{\frac{1}{n}}(n+1)}\right]\left[\left(\frac{e^{r H}-1}{e^{r T}-1}\right)\right]=0
\end{aligned}
$$

Solving the equations (13) and (14) simultaneously, using numerical methods one can get the optimum cycle length $\mathrm{T}=\mathrm{T}_{1}$ and selling price $\mathrm{p}=\mathrm{p}_{1}$. Substituting the optimal values of $\mathrm{T}_{1}$ and $\mathrm{p}_{1}$ in 
equation (12), the optimal value of the net profit is obtained as

$$
\begin{aligned}
& \mathrm{NP}\left(p_{1}, T_{1}\right)=\left(p_{1} e^{r T_{1}}-C e^{r T_{1}}\right)\left(a-\frac{b 1 \theta}{n T}\right) \\
& -\frac{1}{\mathrm{~T}_{1}}\left[\mathrm{~A}+\mathrm{C}\left[\int_{0}^{\mathrm{T}_{1}}\left[\mathrm{a}-b_{1}\left[\frac{\theta t^{\frac{1}{n}-1}}{n T^{\frac{1}{n}}}\right]\right] e^{\left.\beta t^{\alpha} d t-a \mathrm{~T}_{1}+b \theta\right]}\right.\right. \\
& +\mathrm{Ch}\left[\int_{\mathrm{M}}^{\mathrm{T}_{1}} e^{-\beta t^{\alpha}}\left[\int_{\mathrm{t}}^{\mathrm{T}_{1}}\left[\mathrm{a}-b_{1}\left[\frac{\theta t^{\frac{1}{n}-1}}{n T^{\frac{1}{n}}}\right]\right] e^{\beta t^{\alpha}} d t\right] \mathrm{dt}\right] \\
& +\mathrm{CI}_{\mathrm{C}}\left[\int_{\mathrm{M}}^{\mathrm{T}_{1}} e^{-\beta t^{\alpha}}\left[\int_{\mathrm{t}}^{\mathrm{T}_{1}}\left[\mathrm{a}-b_{1}\left[\frac{\theta t^{\frac{1}{n}-1}}{n T^{\frac{1}{n}}}\right]\right] e^{\beta t^{\alpha}} d t\right] \mathrm{dt}\right] \\
& +p_{1} \mathrm{I}_{\mathrm{e}}\left[\frac{\mathrm{aM}^{2}}{2}-\frac{\mathrm{b}_{1} \theta \mathrm{M}^{\frac{\mathrm{n}+1}{\mathrm{n}}}}{T_{1}^{\frac{1}{n}}(n+1)}\right]\left[\frac{e^{r H}-1}{e^{r T_{1}}-1}\right]
\end{aligned}
$$

\section{Case-2: In this case, there is no interest charged} $\boldsymbol{M}>T$.

The interest earned per unit time is

$$
\begin{aligned}
& I E_{2}=p I_{C}\left[\int_{0}^{T} R(t) t d t+R(t)[T(M-T)]\right]\left[\frac{e^{r H}-1}{e^{r T}-1}\right] \\
& =p I_{e}\left[\frac{\mathrm{aT}^{2}}{2}-b_{1} \theta \mathrm{T}+\left[\mathrm{a}-b_{1}\left[\frac{\theta t^{\frac{1}{n}-1}}{n T^{\frac{1}{n}}}\right]\right][T(M-T)]\right]\left[\frac{e^{r H}-1}{e^{r T}-1}\right] \\
& =p I_{e}\left[\frac{\mathrm{aT}^{2}}{2}-b_{1} \theta \mathrm{T}+\left[\mathrm{a}-\frac{b_{1} \theta}{n T}\right][T(M-T)]\right]\left[\frac{e^{r H}-1}{e^{r T}-1}\right] \\
& =p I_{e}\left[\mathrm{aTM}-\frac{\mathrm{aT}^{2}}{2}-\frac{b_{1} \theta \mathrm{M}}{\mathrm{n}}+\left[\frac{1-n}{\mathrm{n}}\right] b_{1} \theta \mathrm{T}\right]\left[\frac{e^{r H}-1}{e^{r T}-1}\right]
\end{aligned}
$$

The total cost per unit time over $(\mathrm{O}, \mathrm{H})$ $\mathrm{TC}=\mathrm{OC}+\mathrm{CD}+\mathrm{IHC}-\mathrm{IE}_{2}$

Using the equations (6), (7), (8) and (15), one can get

$$
\begin{aligned}
& T C=\frac{1}{\mathrm{~T}}\left[\mathrm{~A}+\mathrm{C}\left[\int_{0}^{\mathrm{T}}\left[\mathrm{a}-b_{1}\left[\frac{\theta t^{\frac{1}{n}-1}}{n T^{\frac{1}{n}}}\right]\right] e^{\beta t^{\alpha}} d t-a T+b_{1} \theta\right]\right. \\
& +\mathrm{Ch}\left[\int_{0}^{\mathrm{T}} e^{-\beta t^{\alpha}}\left[\int_{\mathrm{t}}^{\mathrm{T}}\left[\mathrm{a}-b_{1}\left[\frac{\theta t^{\frac{1}{n}-1}}{n T^{\frac{1}{n}}}\right]\right] e^{\beta t^{\alpha}} d t\right] \mathrm{dt}\right] \\
& -p I_{e}\left[\mathrm{aTM}-\frac{\mathrm{aT}^{2}}{2}-\frac{b_{1} \theta \mathrm{M}}{\mathrm{n}}+\left[\frac{1-n}{\mathrm{n}}\right] b_{1} \theta \mathrm{T}\right]\left[\frac{e^{r H}-1}{e^{r T}-1}\right]
\end{aligned}
$$

The net profit is the difference of gross revenue and total revenue cost. The gross revenue is

$$
\left(p e^{r T}-C e^{r T}\right)\left(a-\frac{b_{1} \theta}{n T}\right)
$$

Therefore, net profit is

$$
\begin{aligned}
& N P(p, T)=\left(p e^{r T}-C e^{r T}\right)\left(a-\frac{b_{1} \theta}{n T}\right) \\
& -\frac{1}{\mathrm{~T}}\left[\mathrm{~A}+\mathrm{C}\left[\int_{0}^{\mathrm{T}}\left[\mathrm{a}-b_{1}\left[\frac{\theta t^{\frac{1}{n}-1}}{n T^{\frac{1}{n}}}\right]\right] e^{\beta t^{\alpha}} d t-a T+b_{1} \theta\right]\right.
\end{aligned}
$$

$$
\begin{aligned}
& +\mathrm{Ch}\left[\int_{0}^{\mathrm{T}} e^{-\beta t^{\alpha}}\left[\int_{\mathrm{t}}^{\mathrm{T}}\left[\mathrm{a}-b_{1}\left[\frac{\theta t^{\frac{1}{n}-1}}{n T^{\frac{1}{n}}}\right]\right] e^{\beta t^{\alpha}} d t\right] \mathrm{dt}\right] \\
& +\mathrm{CI}_{c}\left[\int_{0}^{\mathrm{T}} e^{-\beta t^{\alpha}}\left[\int_{\mathrm{t}}^{\mathrm{T}}\left[\mathrm{a}-b_{1}\left[\frac{\theta t^{\frac{1}{n}-1}}{n T^{\frac{1}{n}}}\right]\right] e^{\beta t^{\alpha}} d t\right] \mathrm{dt}\right. \\
& -p I_{e}\left[\mathrm{aTM}-\frac{\mathrm{aT}^{2}}{2}-\frac{b_{1} \theta \mathrm{M}}{\mathrm{n}}+\left[\frac{1-n}{\mathrm{n}}\right] b_{1} \theta \mathrm{T}\right]\left[\frac{e^{r H}-1}{e^{r T}-1}\right]
\end{aligned}
$$

where, $\mathrm{TC}(\mathrm{p}, \mathrm{T})$ is as given in equation (17)

For obtaining the optimal pricing policies of the model one has to maximize $\mathrm{NP}(\mathrm{p}, \mathrm{T})$ with respect to $\mathrm{T}$ and $\mathrm{p}$. The conditions for obtaining optimality are:

$$
\begin{gathered}
\frac{\partial N P(p, T)}{\partial p}=0, \quad \frac{\partial N P(p, T)}{\partial T}=0 \\
\text { and } \mathrm{D}=\left|\begin{array}{ll}
\frac{\partial^{2} N P(p, T)}{\partial p^{2}} & \frac{\partial^{2} N P(p, T)}{\partial p \partial T} \\
\frac{\partial^{2} N P(p, T)}{\partial p \partial T} & \frac{\partial^{2} N P(p, T)}{\partial T^{2}}
\end{array}\right|<0
\end{gathered}
$$

where, D is determinant of the Hessian Matrix

$$
\begin{aligned}
& \frac{\partial N P(p, T)}{\partial T}=0 \text { Implice } \\
& (p-C) r e^{r T}\left[a-\frac{b_{1} \theta}{n T}\right]+(p-C) e^{r T}\left(\frac{b_{1} \theta}{n T^{2}}\right) \\
& +\frac{1}{\mathrm{~T}^{2}}\left[\mathrm{~A}+\mathrm{C}\left[\int_{0}^{\mathrm{T}}\left[\mathrm{a}-b_{1}\left[\frac{\theta t^{\frac{1}{n}-1}}{n T^{\frac{1}{n}}}\right]\right] e^{\beta t^{\alpha}} d t-a T+b_{1} \theta\right]\right. \\
& +\mathrm{Ch}\left[\int_{0}^{\mathrm{T}} e^{-\beta t^{\alpha}}\left[\int_{\mathrm{t}}^{\mathrm{T}}\left[\mathrm{a}-b_{1}\left[\frac{\theta t^{\frac{1}{n}-1}}{n T^{\frac{1}{n}}}\right]\right] e^{\beta t^{\alpha}} d t\right] d t\right] \\
& \left.-\mathrm{pI}_{e}\left[\mathrm{aTM}-\frac{\mathrm{aT}^{2}}{2}-\frac{b_{1} \theta M}{\mathrm{n}}+\left(\frac{1-\mathrm{n}}{\mathrm{n}}\right) b_{1} \theta T\right]\right]\left[\frac{e^{r H}-1}{e^{r T}-1}\right] \\
& -\frac{1}{\mathrm{~T}}\left[\mathrm{~A}+\mathrm{C}\left[\int_{0}^{\mathrm{T}}\left[\mathrm{a}-b_{1}\left[\frac{\theta t^{\frac{1}{n}-1}}{n T^{\frac{1}{n}}}\right]\right] e^{\beta t^{\alpha}} d t-a T+b_{1} \theta\right]\right. \\
& +\mathrm{Ch}\left[\int_{0}^{\mathrm{T}} e^{-\beta t^{\alpha}}\left[\int_{\mathrm{t}}^{\mathrm{T}}\left[\mathrm{a}-b_{1}\left[\frac{\theta t^{\frac{1}{n}-1}}{n T^{\frac{1}{n}}}\right]\right] e^{\beta t^{\alpha}} d t\right] \mathrm{dt}\right] \\
& \left.-\mathrm{pI}_{\mathrm{e}}\left[\mathrm{aTM}-\frac{\mathrm{aT}^{2}}{2}-\frac{b_{1} \theta M}{\mathrm{n}}+\left(\frac{1-\mathrm{n}}{\mathrm{n}}\right) b_{1} \theta T\right]\right] \frac{\mathrm{r}^{r T}\left(e^{r H}-1\right)}{\left(e^{r T}-1\right)} \\
& -\frac{1}{\mathrm{~T}}\left[\mathrm{C}\left[\left[\mathrm{a}-\frac{\mathrm{b}_{1} \theta}{\mathrm{nT}}\right] \mathrm{e}^{\beta t^{\alpha}}-\mathrm{a}+\int_{0}^{\mathrm{T}} \frac{\mathrm{b}_{1} \theta \mathrm{t}^{\frac{1}{n}-1}}{\mathrm{n}^{2} \mathrm{~T}^{\frac{1}{n}+1}} \mathrm{e}^{\beta t^{\alpha}} \mathrm{dt}\right]\right. \\
& +\mathrm{Ch}\left[\left[\mathrm{a}-\frac{b_{1} \theta}{n T}\right] e^{\beta t^{\alpha}} \int_{0}^{\mathrm{T}} e^{-\beta t^{\alpha}} \mathrm{dt}+\left[\int_{0}^{\mathrm{T}} e^{-\beta t^{\alpha}}\left[\int_{t}^{T} \frac{b_{1} \theta t^{\frac{1}{n}-1}}{n^{2} T^{\frac{n+1}{n}}} e^{\beta t^{\alpha}}\right] d t\right] \mathrm{dt}\right] \\
& \left.-\mathrm{pI}_{\mathrm{e}}\left[\mathrm{aM}-\mathrm{aT}+\left(\frac{1-\mathrm{n}}{\mathrm{n}}\right) b_{1} \theta\right]\right]\left[\frac{e^{r H}-1}{e^{r T}-1}\right]=0
\end{aligned}
$$


$\frac{\partial N P(p, T)}{\partial p}=0 \quad$ Implies

$\mathrm{e}^{\mathrm{rT}}\left[\mathrm{a}-\frac{\mathrm{b}_{1} \theta}{\mathrm{nT}}\right]+\frac{\mathrm{I}_{\mathrm{c}}}{\mathrm{T}}\left[\mathrm{aTM}-\frac{\mathrm{aT}^{2}}{2}-\frac{b_{1} \theta M}{\mathrm{n}}+\left(\frac{1-\mathrm{n}}{\mathrm{n}}\right) b_{1} \theta T\right]\left[\frac{e^{r H}-1}{e^{r T}-1}\right]=0$

Solving the equations (19) and (20) simultaneously using numerical methods for given values of parameters, one can get the optimum cycle length $\mathrm{T}=\mathrm{T}_{2}$ and selling price $\mathrm{p}=\mathrm{p}_{2}$. Substituting the optimal value of $\mathrm{T}_{2}$ and $\mathrm{p}_{2}$ in equation (18), one can get the optimal value of the net profit as

$$
\begin{aligned}
& N P^{*}\left(p_{2}, T_{2}\right)=\left(p_{2} e^{r T_{2}}-C e^{r T_{2}}\right)\left[\mathrm{a}-\frac{\mathrm{b}_{1} \theta}{\mathrm{n} T_{2}}\right] \\
- & \frac{1}{T_{2}}\left[\mathrm{~A}+\mathrm{C}\left[\int_{0}^{T_{2}}\left[\mathrm{a}-b_{1}\left[\frac{\theta t^{\frac{1}{n}-1}}{n T_{2}^{\frac{1}{n}}}\right]\right] e^{\beta t^{\alpha}} d t-a T_{2}+b_{1} \theta\right]\right. \\
+ & \mathrm{Ch}\left[\int_{\mathrm{m}}^{T_{2}} e^{-\beta t^{\alpha}}\left[\int_{\mathrm{t}}^{T_{2}}\left[\mathrm{a}-\frac{b_{1} \theta t^{\frac{1}{n}-1}}{n T_{2^{\frac{1}{n}}}^{\frac{1}{n}}}\right] e^{\beta t^{\alpha}} d t\right] \mathrm{dt}\right] \\
- & \mathrm{p}_{2} \mathrm{I}_{\mathrm{e}}\left[\frac{\mathrm{am}^{2}}{2}-\frac{\mathrm{b}_{1} \theta \mathrm{m}^{\frac{\mathrm{n}+1}{\mathrm{n}}}}{T_{2} \frac{1}{n}(n+1)}\right]\left[\frac{e^{r H}-1}{e^{r T_{2}-1}}\right]
\end{aligned}
$$

\section{NUMERICAL ILLUSTRATION:}

Consider the case of deriving the economic order quantity and other optimal ordering policies for markets dealing with deteriorating products. From the records the values of the parameters can be estimated. The deteriorating parameters $\alpha, \beta$ are estimated to vary from 0.6 to 0.68 and 0.061 to 0.068 respectively. The values of other parameters are $a=2100$ units, $\mathrm{b}_{1}=20$ units, $\mathrm{C}=$ Rs.2.0, $\mathrm{h}=$ Rs.0.15, $\mathrm{I}_{\mathrm{c}}=$ Rs.0.1, $\mathrm{I}_{\mathrm{e}}=$ Rs. 0.16, $\mathrm{H}=12$ months, $\mathrm{M}$ $=0.5$ months, $\mathrm{r}=0.08, \mathrm{~A}=\mathrm{Rs} .2500, \mathrm{n}=0.6, \theta=$ 50 . The optimal value of selling price (p) and cycle length $(\mathrm{T})$ are obtained by using the equation (13) and (14) or (19) and (20). The optimal values of T are taken as $\mathrm{T}=T_{1}$ if $\mathrm{M} \leq \mathrm{T}_{1}$ and $\mathrm{T}=\mathrm{T}_{2}$ if $\mathrm{M}>T_{1}$. By substituting these values of cycle time $\mathrm{T}$ and selling price $\mathrm{p}$ the optimal values of Order quality $\mathrm{Q}$ and net profit NP are obtained and presented in Table-1.

From Table-1, it is observed that when the parameter ' $a$ ' increases from 2100 units to 2400 units then the optimal order quantity ' $Q$ ' is increasing from 451.75 units to 603.84 units, the net profit 'NP' per unit time is increasing from Rs.1607 to Rs.1962 and the optimal selling price ' $p$ ' is increasing from Rs.93.45 to 94.51 and the optimal cycle length ' $\mathrm{T}$ ' is decreasing from 0.56 to 0.54 when other parameters and cost are fixed. If the parameter ' $b_{1}$ ' is increasing from 21 to 23 then the optimal order quantity ' $Q$ ' is increasing from 451.75 units to 582.89 units, the net profit 'NP' per unit time is increasing from Rs.1607 to Rs.1962 and the optimal selling price is increasing from Rs.87.46 to Rs.88.96 are increasing and the optimal cycle length ' $\mathrm{T}$ ' is increasing from 0.55 to 0.56 when other parameters cost are fixed.

As the deterioration parameter ' $\alpha$ ' is increasing from 0.62 to 0.68 then the optimal order quantity ' $Q$ ' is decreasing from 506.76 units to 494.82 units, the selling price ' $p$ ' is decreasing from Rs.92.32 to Rs.91.52, the net profit 'NP' per unit time is increasing from Rs.1648 to Rs.1667 and no change in the optimal cycle length ' $T$ '. When the parameters ' $\beta$ ' is increasing from 0.64 to 0.68 then the optimal order quantity ' $Q$ ' is increasing from 501.90 units to 508.63 units, the net profit 'NP' per unit time is increasing from Rs.1652 to Rs.1680 and the selling price 'p' is decreasing from Rs.92.90 to Rs.92.52 and the optimal cycle length ' $\mathrm{T}$ ' is increasing from 0.57 to 0.59 for fixed values of other parameters and costs. When the unit cost ' $\mathrm{C}$ ' is increasing from Rs.2.0 to Rs.2.4 then the optimal order quantity ' $Q$ ' is decreasing from 513.64 units to 513.34 units, the net profit 'NP' per unit time is increasing from Rs.1641 to Rs.1675 and the selling price ' $p$ ' is increasing form Rs.91.22 to Rs.92.76 and the optimal cycle length ' $\mathrm{T}$ ' is increasing from 0.56 to 0.57 when other parameters and costs are fixed.

When holding cost ' $h$ ' is increasing from Rs.0.51 to Rs.0.53 then the optimal order quantity ' $Q$ ' is remains the same, the selling price ' $p$ ' is decreasing from Rs.92.20 to Rs.92.17, the net profit 'NP' per unit time is increasing from Rs.1794 to Rs.1796 and no change in the optimal cycle length ' $\mathrm{T}$ '. The interest changed ' $\mathrm{I}_{c}$ ' increases from Rs.0.611 to Rs.0.613 then the optimal order quantity ' $Q$ ' is remains the same, the selling price ' $p$ ' is increasing from Rs.91.30 to Rs.91.50, the net profit 'NP' per unit time is decreasing from Rs.1805 to Rs.1802 and the optimal cycle length ' $\mathrm{T}$ ' is increasing from 0.54 to 0.56 when other parameters and costs are fixed. The unit earned ' $\mathrm{I}_{e}$ ' increases from Rs.0.161 to Rs.0.163 then the optimal order quantity ' $Q$ ' is increasing from 526.34 8units to 526.68 units and the net profit 'NP' per unit time is increasing from Rs.1810 to Rs.1825, the selling price ' $p$ ' is decreasing from Rs.90.51 to Rs.88.60 and no change in the optimal cycle length ' $T$ '. When the time horizon ' $H$ ' increases from 12.5 months to 13.5 months then the optimal order quantity ' $Q$ ' is increasing from 520.37 units to 524.76 units, the net profit 'NP' per unit time is increasing from Rs.1608 to Rs.1665, the unit selling price ' $p$ ' is decreasing from Rs.92.86 to Rs.92.64 and the optimal cycle length ' $\mathrm{T}$ ' is increasing from 0.54 to 0.57 . 
International Journal of Research in Advent Technology, Vol.7, No.1, January 2019

E-ISSN : 2321-9637

Available online at www.ijrat.org

Table-1

Values of T, p, Q and NP for different values of parameters

\begin{tabular}{|c|c|c|c|c|c|c|c|c|c|c|c|c|c|c|c|c|c|}
\hline$a$ & $\mathrm{~b}$ & $\mathrm{C}$ & $\alpha$ & $\beta$ & $\mathrm{r}$ & $\mathrm{h}$ & $\mathrm{I}_{\mathrm{c}}$ & $\overline{I_{e}}$ & $\mathrm{~A}$ & $\mathrm{H}$ & $\mathrm{n}$ & $\mathrm{M}$ & $\theta$ & $\mathrm{T}$ & $\mathrm{p}$ & $\mathrm{Q}$ & NP \\
\hline 2100 & 20 & 2.0 & 0.6 & 0.02 & 0.08 & 0.1 & 0.15 & 0.12 & 2500 & 12 & 0.6 & 0.5 & 50 & 0.56 & 93.45 & 451.75 & 1607 \\
\hline 2200 & & & & & & & & & & & & & & 0.55 & 93.56 & 518.40 & 1791 \\
\hline 2300 & & & & & & & & & & & & & & 0.54 & 94.09 & 582.89 & 1709 \\
\hline \multirow[t]{40}{*}{2400} & & & & & & & & & & & & & & 0.54 & 94.51 & 603.84 & 1962 \\
\hline & 21 & & & & & & & & & & & & & 0.55 & 87.46 & 451.75 & 1607 \\
\hline & 22 & & & & & & & & & & & & & 0.55 & 88.51 & 525.43 & 1861 \\
\hline & 23 & & & & & & & & & & & & & 0.56 & 88.96 & 582.89 & 1962 \\
\hline & & 2.2 & & & & & & & & & & & & 0.56 & 91.22 & 513.64 & 1641 \\
\hline & & 2.4 & & & & & & & & & & & & 0.57 & 92.76 & 513.34 & 1675 \\
\hline & & & 0.62 & & & & & & & & & & & 0.56 & 92.32 & 506.76 & 1648 \\
\hline & & & 0.64 & & & & & & & & & & & 0.56 & 91.93 & 500.97 & 1654 \\
\hline & & & 0.66 & & & & & & & & & & & 0.56 & 91.59 & 499.61 & 1660 \\
\hline & & & 0.68 & & & & & & & & & & & 0.56 & 91.52 & 494.82 & 1667 \\
\hline & & & & 0.021 & & & & & & & & & & 0.57 & 92.90 & 501.90 & 1652 \\
\hline & & & & 0.022 & & & & & & & & & & 0.58 & 92.52 & 506.51 & 1661 \\
\hline & & & & 0.023 & & & & & & & & & & 0.59 & 92.07 & 508.63 & 1680 \\
\hline & & & & & 0.082 & & & & & & & & & 0.56 & 92.86 & 520.37 & 1608 \\
\hline & & & & & 0.084 & & & & & & & & & 0.56 & 92.64 & 523.07 & 1736 \\
\hline & & & & & 0.086 & & & & & & & & & 0.56 & 92.43 & 524.76 & 1765 \\
\hline & & & & & 0.088 & & & & & & & & & 0.56 & 92.21 & 528.41 & 1794 \\
\hline & & & & & & 0.11 & & & & & & & & 0.56 & 92.20 & 528.41 & 1794 \\
\hline & & & & & & 0.12 & & & & & & & & 0.56 & 92.18 & 528.41 & 1794 \\
\hline & & & & & & 0.13 & & & & & & & & 0.56 & 92.17 & 528.41 & 1796 \\
\hline & & & & & & & 0.16 & & & & & & & 0.54 & 91.30 & 525.51 & 1805 \\
\hline & & & & & & & 0.17 & & & & & & & 0.55 & 91.40 & 525.51 & 1804 \\
\hline & & & & & & & 0.18 & & & & & & & 0.56 & 91.50 & 525.51 & 1802 \\
\hline & & & & & & & & 0.13 & & & & & & 0.56 & 90.51 & 526.34 & 1810 \\
\hline & & & & & & & & 0.14 & & & & & & 0.56 & 89.54 & 526.51 & 1818 \\
\hline & & & & & & & & 0.15 & & & & & & 0.56 & 88.60 & 526.68 & 1825 \\
\hline & & & & & & & & & 2600 & & & & & 0.57 & 93.23 & 534.20 & 1879 \\
\hline & & & & & & & & & 2650 & & & & & 0.58 & 94.86 & 525.73 & 1933 \\
\hline & & & & & & & & & & 13 & & & & 0.54 & 92.86 & 520.37 & 1608 \\
\hline & & & & & & & & & & 14 & & & & 0.57 & 92.64 & 524.76 & 1665 \\
\hline & & & & & & & & & & & 0.61 & & & 0.59 & 92.26 & 524.48 & 1811 \\
\hline & & & & & & & & & & & 0.62 & & & 0.53 & 92.37 & 514.37 & 1826 \\
\hline & & & & & & & & & & & 0.63 & & & 0.49 & 92.51 & 498.14 & 1837 \\
\hline & & & & & & & & & & & 0.64 & & & 0.46 & 92.73 & 475.86 & 1891 \\
\hline & & & & & & & & & & & & 0.51 & & 0.55 & 92.14 & 582.16 & 1964 \\
\hline & & & & & & & & & & & & 0.52 & & 0.55 & 92.36 & 582.21 & 1927 \\
\hline & & & & & & & & & & & & 0.53 & & 0.56 & 92.82 & 582.37 & 1832 \\
\hline & & & & & & & & & & & & & 51 & 0.55 & 93.52 & 525.34 & 1689 \\
\hline & & & & & & & & & & & & & 52 & 0.56 & 92.83 & 525.81 & 1824 \\
\hline & & & & & & & & & & & & & 53 & 0.56 & 92.76 & 526.83 & 1937 \\
\hline
\end{tabular}

The permissible delay period ' $\mathrm{M}$ ' increases from 0.52 months to 0.54 months then the optimal order quantity ' $Q$ ' is increasing from 582.16 units to 582.37 units, the net profit 'NP' per unit time is decreasing from Rs.1964 to Rs.1832, the optimal selling price ' $p$ ' is increasing from Rs.91.47 to Rs.92.82 and the optimal cycle length ' $\mathrm{T}$ ' is increasing from 0.55 to 0.56 . If the inflation rate ' $r$ ' increases from Rs.0.082 to Rs.0.088 then the optimal order quantity ' $Q$ ' is increasing from 520.37 units to 528.41 units, the net profit 'NP' per unit time is increasing from Rs.1608 to Rs.1694, the unit selling price ' $p$ ' is decreasing from Rs.92.86 to Rs.92.21 and the optimal cycle length
' $\mathrm{T}$ ' is also increasing from 0.56 to 0.56 when other parameter and costs are fixed.

If the ordering cost ' $A$ ' increases from Rs.2600 to Rs.2650 then the optimal order quality ' $Q$ ' is increasing from 526.20 units to 527.73 units, the net 'NP' per unit time is decreasing from Rs. 1779 to Rs. 1608 , and the unit selling price ' $p$ ' is increasing from Rs.91.23 to Rs.93.86 and the optimal cycle length ' $\mathrm{T}$ ' is increasing from 0.57 to 0.58 when other parameter and cost are fixed. If the total demand during the cycle period ' $\theta$ ' increases from 51 to 53 then the optimal order quantity ' $Q$ ' is increasing from 589.41 units to 598.34 units, the 
net profit 'NP' per unit time is increasing from Rs.1689 to Rs.1937, and the unit selling price ' $p$ ' is decreasing from Rs.93.52 to Rs.92.76 and the optimal cycle length ' $\mathrm{T}$ ' is increasing from 0.54 to 0.59 when other parameters and costs are fixed.

If the demand index parameter ' $n$ ' increases from 0.61 to 0.64 , then the optimal order quantity ' $\mathrm{Q}$ ' is decreasing from 524.48 units to 475.86 units, the net profit 'NP' per unit time is increasing from Rs.1811 to Rs.1891, and the unit selling price ' $p$ ' is increasing from Rs.92.26 to Rs.92.73 and the optimal cycle length ' $\mathrm{T}$ ' is decreasing from Rs.0.59 to Rs.0.46 when other parameters and costs are fixed.

\section{SENSITIVITY ANALYSIS:}

To study the effect of changes in the model parameters and costs on the optimal values of the cycle length and selling price, the sensitivity analysis is carried. The following data on parameters and costs is used: $\mathrm{a}=2100$ units, $\mathrm{b}_{1}=20$ units, $\mathrm{C}=$ Rs. $2.0, \mathrm{~h}=$ Rs.0.15, $\mathrm{I}_{c}=$ Rs. 0.1, $\mathrm{I}_{e}=R s .0 .16, \mathrm{H}=12$ months, $\mathrm{M}=0.5$ months, $\mathrm{r}=0.08, \mathrm{~A}=\mathrm{Rs} .2500, \mathrm{n}=0.6, \theta=50$. Sensitivity analysis of the model is performed with respect to cost $A$, unit cost $C$, holding cost $h$, carrier function, demand parameters $\mathrm{a}$ and $\mathrm{b}$. Inflation parameter $r$ and all parameter together on ordering quantity, selling price, cycle length and net profit. Table 2 and 3 summarize the variations in $\mathrm{T}, \mathrm{p}, \mathrm{Q}, \mathrm{NP}$ for $-15 \%,-10 \%,-5 \%, 5 \%, 10 \%$, $15 \%$ variation of the parameter and costs. The relationship between the parameter and the optimal values are shown Figure-2.

The performance measure of the model is highly affected by demand parameters, ' $a$ ' and ' $b$ '. As ' $a$ ' increases the optimal order quantity ' $Q$ ' is decreasing, the net profit 'NP' and the optimal selling price ' $p$ ' are increasing. Similarly, the parameter ' $b$ ' is increasing the selling price ' $p$ ', the net profit 'NP' are decreasing, and the optimal order quantity ' $\mathrm{Q}$ ' is increasing.

It is further observed that there is a moderate influence of the unit cost ' $\mathrm{C}$ ' on the optimal values of ' $T$ ' and ' $p$ '. As ' $C$ ' increases the optimal order quantity ' $Q$ ' is remains the same, the selling price ' $p$ ' and the net profit 'NP' are increasing. Similarly, the rate of inflation ' $r$ ' is increasing the optimal order quantity ' $Q$ ', the net profit 'NP' and the selling price ' $p$ ' are increasing. It is further observed that there is moderate influence of the interest changed ' $\mathrm{I}_{c}$ ' and interest earned ' $\mathrm{I}_{e}$ ' the optimal values of ' $\mathrm{T}$ ' and ' $\mathrm{p}$ '. As ' $\mathrm{I}_{c}$ ' increases the optimal order quantity ' $\mathrm{Q}$ ' is remains the same, the selling price ' $p$ ' is decreasing, and the net profit 'NP' is increasing, the net profit 'NP' and the selling price ' $p$ ' are decreasing.
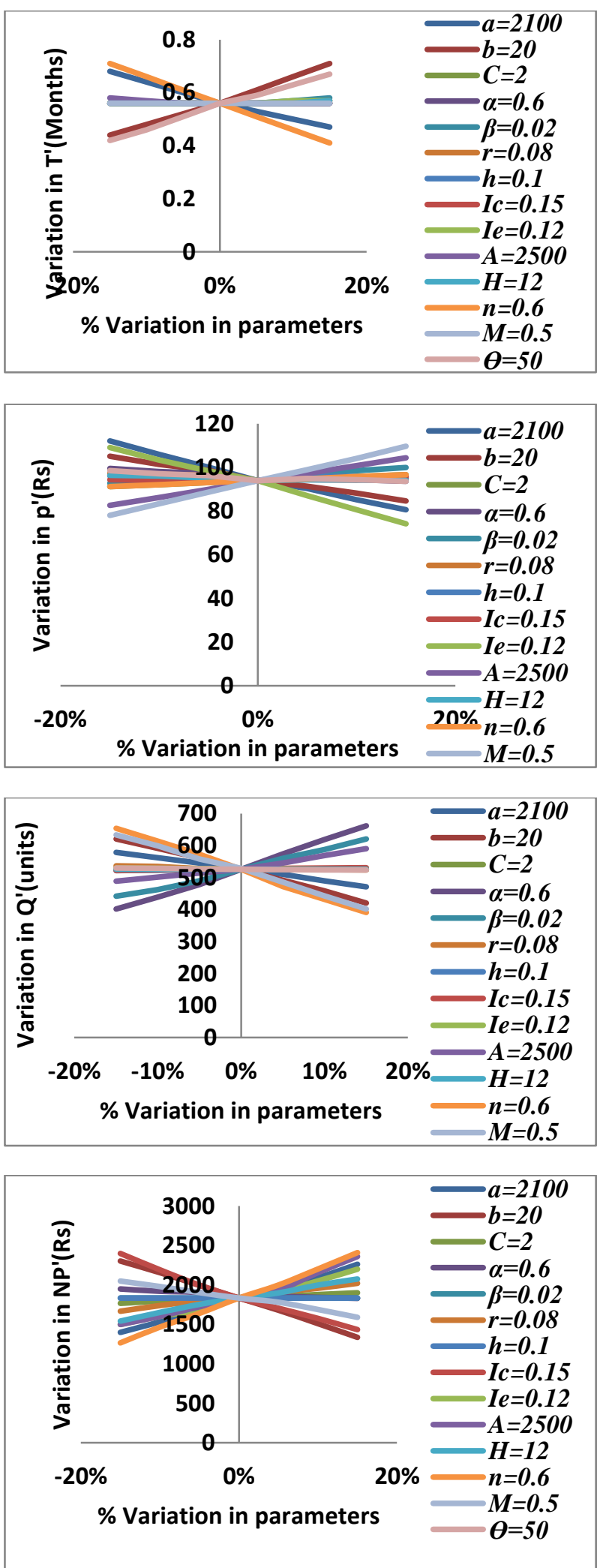

Figure-2 Graphical representation of the sensitivity analysis of the inventory model with dependent demand. 
International Journal of Research in Advent Technology, Vol.7, No.1, January 2019

E-ISSN: 2321-9637

Available online at www.ijrat.org

Figure-2

Sensitive Analysis of the model

\begin{tabular}{|c|c|c|c|c|c|c|c|c|}
\hline \multirow{2}{*}{\multicolumn{2}{|c|}{$\begin{array}{l}\text { Variation } \\
\text { Parameters }\end{array}$}} & \multicolumn{7}{|c|}{ Percentage Change in Parameters } \\
\hline & & -15 & -10 & -5 & 0 & 5 & 10 & 15 \\
\hline \multirow{3}{*}{ a } & $\mathrm{T}$ & 0.68 & 0.64 & 0.6 & 0.56 & 0.53 & 0.5 & 0.47 \\
\hline & $\mathrm{P}$ & 112.08 & 104.86 & 100 & 93.86 & 90.33 & 85.15 & 80.51 \\
\hline & $\mathrm{NP}$ & 1398 & 1534 & 1707 & 1833 & 1962 & 2092 & 2261 \\
\hline \multirow{2}{*}{$\mathrm{b}$} & $\mathrm{T}$ & 0.44 & 0.48 & 0.52 & 0.56 & 0.61 & 0.66 & 0.71 \\
\hline & $\mathrm{NP}$ & 2302 & 2155 & 1994 & 1833 & 1679 & 1509 & 1335 \\
\hline \multirow{4}{*}{$\alpha$} & $\mathrm{T}$ & 0.56 & 0.56 & 0.56 & 0.56 & 0.56 & 0.56 & 0.56 \\
\hline & $\mathrm{P}$ & 99.42 & 98.04 & 97.23 & 93.86 & 94.9 & 94.28 & 93.66 \\
\hline & $\mathrm{Q}$ & 526.23 & 526.06 & 525.89 & 525.73 & 525.56 & 525.4 & 525.23 \\
\hline & NP & 1766 & 1788 & 1733 & 1833 & 1855 & 1877 & 1900 \\
\hline \multirow{4}{*}{$\mathrm{C}$} & $\mathrm{T}$ & 0.56 & 0.56 & 0.56 & 0.56 & 0.56 & 0.57 & 0.58 \\
\hline & $\mathrm{P}$ & 91.11 & 92.03 & 92.95 & 93.86 & 94.77 & 95.68 & 96.59 \\
\hline & $\mathrm{Q}$ & 442.18 & 462.75 & 489.65 & 525.73 & 560.3 & 587.77 & 620.3 \\
\hline & NP & 1833 & 1833 & 1833 & 1833 & 1833 & 1833 & 1833 \\
\hline \multirow{4}{*}{$\mathrm{r}$} & $\mathrm{T}$ & 0.56 & 0.56 & 0.56 & 0.56 & 0.56 & 0.56 & 0.56 \\
\hline & $\mathrm{P}$ & 97.15 & 96.7 & 96.33 & 93.86 & 95.67 & 95.66 & 95.76 \\
\hline & $\mathrm{Q}$ & 536.98 & 534.32 & 528.06 & 525.73 & 525.34 & 524.97 & 524.45 \\
\hline & $\mathrm{NP}$ & 1666 & 1738 & 1797 & 1833 & 1906 & 1957 & 2020 \\
\hline \multirow{2}{*}{$\mathrm{h}$} & $\mathrm{T}$ & 0.56 & 0.56 & 0.56 & 0.56 & 0.56 & 0.56 & 0.56 \\
\hline & $\mathrm{P}$ & 95.98 & 95.94 & 95.9 & 93.86 & 95.83 & 95.79 & 95.03 \\
\hline \multirow{3}{*}{$\mathrm{I}_{\mathrm{e}}$} & $\mathrm{P}$ & 109 & 103.36 & 96.33 & 93.86 & 85.99 & 79.61 & 74.09 \\
\hline & $\mathrm{Q}$ & 529.56 & 528.9 & 528.23 & 525.73 & 526.89 & 526.23 & 525.57 \\
\hline & NP & 1498 & 1601 & 1686 & 1833 & 1915 & 2050 & 2199 \\
\hline \multirow{4}{*}{ A } & $\mathrm{T}$ & 0.58 & 0.57 & 0.56 & 0.56 & 0.56 & 0.56 & 0.56 \\
\hline & $\mathrm{P}$ & 82.55 & 86.15 & 89.81 & 93.86 & 97.11 & 100.74 & 104.36 \\
\hline & $\mathrm{Q}$ & 488.99 & 501.25 & 513.77 & 525.73 & 545.05 & 568.94 & 590.77 \\
\hline & $\mathrm{NP}$ & 1498 & 1601 & 1723 & 1833 & 1935 & 2155 & 2359 \\
\hline \multirow{4}{*}{$\mathrm{H}$} & $\mathrm{T}$ & 0.56 & 0.56 & 0.56 & 0.56 & 0.56 & 0.56 & 0.57 \\
\hline & $\mathrm{P}$ & 92.67 & 93.63 & 94.7 & 93.86 & 96.96 & 98.47 & 99.91 \\
\hline & $\mathrm{Q}$ & 525.73 & 525.73 & 525.73 & 525.73 & 525.73 & 525.73 & 525.73 \\
\hline & NP & 1541 & 1651 & 1753 & 1833 & 1898 & 1997 & 2074 \\
\hline \multirow{4}{*}{$\mathrm{n}$} & $\mathrm{T}$ & 0.71 & 0.66 & 0.61 & 0.56 & 0.51 & 0.46 & 0.41 \\
\hline & $\mathrm{P}$ & 91.11 & 92.03 & 92.95 & 93.86 & 94.77 & 95.68 & 96.59 \\
\hline & $\mathrm{Q}$ & 653.88 & 614.26 & 572.61 & 525.73 & 472.08 & 432.33 & 390.69 \\
\hline & $\mathrm{NP}$ & 1265 & 1465 & 1639 & 1833 & 1998 & 2201 & 2409 \\
\hline \multirow{4}{*}{ M } & $\mathrm{T}$ & 0.56 & 0.56 & 0.56 & 0.56 & 0.56 & 0.56 & 0.56 \\
\hline & $\mathrm{P}$ & 80.04 & 83.25 & 87.62 & 93.86 & 99.9 & 104 & 109.65 \\
\hline & $\mathrm{Q}$ & 633.14 & 599.26 & 558.07 & 528.73 & 486.18 & 442.75 & 401.65 \\
\hline & $\mathrm{NP}$ & 2048 & 1979 & 1902 & 1833 & 1780 & 1688 & 1589 \\
\hline$\Theta$ & $\mathrm{T}$ & 0.42 & 0.46 & 0.51 & 0.56 & 0.59 & 0.63 & 0.67 \\
\hline
\end{tabular}




\section{CONCLUSIONS}

In this paper an economic order level inventory model under delay in payments and inflation for deteriorating items with power pattern demand is developed and analyzed. It is assumed that the life time of the commodity is random and follows a Weibull distribution. Here it is assumed that the money value changes over time. The power pattern demand includes several forms of demand for different values of the index parameter. The deterioration rate includes increasing /decreasing/ constant rates of deterioration. The optimal ordering and pricing of model are derived by maximizing the profit rate function. The sensitivity analysis of the model reveals that the deterioration and demand parameter have influence on optimal ordering quantities. The rate of inflation is having significant influence on ordering and pricing policies of the model. It is observed that this model serves the purpose of scheduling the orders in market yards dealing with deteriorating items and time dependent demand. It is possible to extend this model for multi commodity EOQ system which will be considered later.

\section{REFERENCES}

[1] Aggaarwal, S.P. "A note of an order level inventory model for a system with constant rate of deterioration", Opsearch, Vol 15(4), 184-187, 1978.

[2] Aggaarwal, S.P. and Jaggi, C.K. "Ordering policies of deteriorating items under permissible delay in payments", journal of the operational research society, 46,658-662, 1995.

[3] Bierman.H, and Thomas. "Inventory Decisions under inflationary conditions", Dec.Sci, 8(1), 151-155, 1977.

[4] Brahmbhatt A.C. "Economic order quantity under variable rate of inflation and mark-up prices", Productivity, 23,127-130, 1982.

[5] Buzacott J.A. "Economic order quantities with inflation, operational research quarterly", Vol.26, Issue 1, 553-558, 1975.

[6] Chung , K.J., Lio, J.J. "Lot sizing decisions under trade credit depending on the order quantity",J.Computers \& Operation Research, Vol.31,909-928, 2004.

[7] Dave, U. and Shah, Y.K. "A probabilistic inventory model for deteriorating items with lead time equal to one scheduling period", European journal of operation research,Vol.9,No.3,281-285, 1982.

[7] Dave , U. and Shah, Y.K. "A probabilistic inventory model for deteriorating items with lead time equal to one scheduling period", European journal of operation research,Vol.9,No.3,281-285, 1982.

[8] Dhir Singh and S.R. Singh. "Review of Literature and survey of the developed inventory models". Int. J. Math. And Appl., 6(1-D) ,673-685, 2018.

[9] Ghare, P.N. and Scrader, G.F. "A model for exponentially decaying inventories", journal of industrial engineering, 15:238-243, 1963.

[10] Misra, R.B. "A note on optimal inventory management under inflation, Naval Res. Logist. Quart. 26 161-165, 1979.

[11] Giri, B.C. and Chaudhuri, K.S. "Deterministic models of perishable inventory with stock dependent demand rate and nonlinear holding cost", European journal of operation research,105:467-474, 1998.

[12] Goyal, V.P. and Aggarwal, S.P. "Pricing and ordering policy with general Weibull rate of deteriorating inventory", Indian Journal of Pure and Applied Mathematics, Vol.11:5,618627, 1980.

[13] Goyal, S.K. and Giri, B.C. "Invited review recent trends in modelling of deteriorating inventory. European Journal of Operational research society, Vol.134,1-16, 2001.

[14] Goyal, S.K. "Economic ordering quantity under conditions of permissible delay in payments, Journal of Operation Research Society, Vol.36, 335-338, 1985.

[15] Horng-Jinh chang, Chung-Yuan Dye. “ An inventory model for deteriorating items under the condition of permissible delay in payments. Yugoslav Journal of Operations Research, 12,73-84, 2002.

[16] H.S.Shukla, R.P.Tripathi, A.Siddiqui and Vivek Shukla. "EOQ model with inventory level dependent demand rate under permissible delay in payments with cash discount. Indian journal of science and technology, Vol 8(28), 2015.

[17] Jyothi, Geetanjali Sharma, Mahesh Kumar Jayaswal. "Inventory model having price dependent demand and permissible delay in payments under learning effect for deteriorating items. international Journal of Computer Engineering \& Technology (IJCET), Vol. 9, Issue 5, pp. 142-151, 2018.

[18] Jui-Jung Liao. "On an EPQ model for deteriorating items under permissible delay in payments", Applied Mathematical Modelling, Volume 31,393-403, 2007.

[19] Kalpakam, S and Sapna, K.P. "An (s, S) perishable system with arbitrary distributed lead times", Opsearch, Vol.33, 1-19, 1996a. 
[20] K.Srinivasa Rao, G.Sridevi and K.Nirupama Devi. "Inventory model for deteriorating items with Weibull rate of replenishment and selling price dependent demand.International journal of Operations Research, 9(3):329-349, 2010.

[21] Liang-Yuh Ouyang, Kun-Shan Wu and ChihTe Yang. "A study on an inventory model for non-instantaneous deteriorating items with permissible delay in payments", Computers \& Industrial Engineering, Volume 51, Issue 4,637-651, 2006.

[22] Namhias, S. "Perishable inventory theory: A review, Operations Research", Vol.30:4, 680708, 1978.

[23] Nurrupama Devi, et al. "Optimal policy and ordering policies for the deteriorating inventory having mixed weibull rate of decay, proceedings of AP Academic of Sciences" Vol.8, No.2, 125-132, 2004.

[24] Ouyang, L. Y., Teng, J. T. and Chen, L. H. "Optimal ordering policy for deteriorating items with partial backlogging under permissible delay in payments", Journal of Global Optimization, Vol.34, No.2, 245, 2006.

[25] Pal, M. “An inventory model for deteriorating items when demand is random", Calcutta Statistical Association Bulletin, Vol.39,201207, 1990.

[26]Raman Patel and D.M. Patel, "Inventory Model with Different Deterioration Rates With Shortages ,Time AND Price Dependent Demand Under Inflation AND Permissible Delay in Payments", Global Journal of Pure and Applied Mathematics, ISSN 0973-1768 Volume 13, Number 6 (2017),pp.1499-1514 Research India Publications, 2017.

[27]R.Amuthaand Dr.E.Chandrasekhararan. "An Inventory Model FOR Constant Demand with Shortages Under Permissible Delay in Payments", JOSR, Jouranal Of Mathematics (IOSR-JM).,e-ISSN:2278-5728,p-ISSN: 2319765X, Volume 6,Issue 5 (May-Jun.2013) PP 28-33, 2013.

[28] Ruxian Li, et al. (2010). A review on deteriorating inventory study, journal of science and management,3: 117-129, 2010.

[29] R.P.Tripathi and Shweta Singh Tomar.,(2016). Optimal Order Policy for deteriorating items with permissible delay in payments and Non-Linear holding cost. International journal of modern mathematical science, 14(3):335-351, 2016.

[30] Ravi Gor and Nita H Shah (2006). An EOQ model for deteriorating items with price dependent demand and permissible delay in payments under inflation. Opsearch, Vol. 43. No.4, 2006.
[31] Sarker, B.R. et al. (2000).Supply chain models for perishable products under inflation and permissible delay in payments, Computers \& Operations Research,27,59-75, 2000.

[32] Shah,Y. and Jaiswal,M.C. (1977). An order level inventory model for a system withconstant rate of deterioration, Opsearch,Vol.14,174-184, 1977.

[33] Shital S.Patel.,(2017). "Inventory model with different deterioration rates for imperfect quality items and inflation considering price and time dependent demand under permissible delay in payments", Int. Jor. of latest technology in engineering, management \& applied science(IJLTEMAS) Vol. VI, Issue VIIIS, 2017.

[34] Sushil Kumar, U.S.Rajput.,(2013). “An Inflationary inventory model for Weibull deteriorating items with constant demand and partial backlogging under permissible delay in payments". American journal of engineering research(AJER)., Vol. 02, Issue- 09, pp-46-54, 2013.

[35] Sanjey Kumar and Neeraj Kumar.(2016). “An inventory model for deteriorating items under inflation and permissible delay in payments by genetic algorithm", cognet business \& management, 3:1239605, 2016.

[36] Tadikamalla, P.R., (1978), “An EOQ model for items with gamma distributed deterioration", AIIE Trans. 10, 100-103, 1978.

[37] Venkata Subbaiah, K., Srinivas Rao, K. Nirupama Devi, K.(2005). "An EOQ model for deteriorating items with stock depending demand Assam Statistical Review", a Research Journal of statistics, Vol.19, No.2, 84-94, 2005. 